\title{
Abatacept might increase bone mineral density at femoral neck for patients with rheumatoid arthritis in clinical practice: AIRTIGHT study
}

Masahiro Tada, Kentaro Inui, Yuko Sugioka, Kenji Mamoto, Tadashi Okano, Tatsuya Koike

\begin{tabular}{|c|l|}
\hline Citation & Rheumatology International. 38(5); 777-784 \\
\hline Issue Date & 2018-01-02 \\
\hline Type & Journal Article \\
\hline Textversion & Author \\
\hline Relation & $\begin{array}{l}\text { This is a post-peer-review, pre-copyedit version of an article published in Rheumatology } \\
\text { International. The final authenticated version is available online at: } \\
\text { https://doi.org/10.1007/s00296-017-3922-z. }\end{array}$ \\
\hline DOI & $10.1007 / \mathrm{s} 00296-017-3922-\mathrm{z}$ \\
\hline
\end{tabular}

Self-Archiving by Author(s)

Placed on: Osaka City University

Tada, M., Inui, K., Sugioka, Y. et al. Abatacept might increase bone mineral density at femoral neck for patients with rheumatoid arthritis in clinical practice: AIRTIGHT study. Rheumatology International. 38, 777-784 (2018). doi:10.1007/s00296-017-3922-z 
Original article

Abatacept might increase bone mineral density at femoral neck for patients with rheumatoid arthritis in clinical practice -AIRTIGHT study- 


\section{ABSTRACT}

Objective: We investigated the influence of abatacept (ABT) on bone mineral density (BMD) and bone metabolic markers (BMMs) in patients with rheumatoid arthritis (RA) compared to other biologic disease-modifying anti-rheumatic drugs (bDMARDs).

Methods: This prospective, comparative, non-randomized study (the AIRTIGHT study; UMIN000005570) investigated the effects of ABT and other bDMARDs on bone metabolism. A total of 165 RA patients were divided into ABT $(n=50)$ and Non-ABT $(n=115)$. We evaluated percentage changes in BMD $(\% \Delta B M D)$ at the lumbar spine and femoral neck using dual-energy X-ray absorptiometry. Urinary levels of cross-linked $\mathrm{N}$-telopeptide of type I collagen (uNTx) and bone-specific alkaline phosphatase (BAP) were used as markers of bone resorption and formation, respectively.

Results: No significant differences in 1-year completion rates were seen between ABT (64\%) and Non-ABT (72\%; $\mathrm{p}=0.387)$. The $\% \triangle \mathrm{BMD}$ at the femoral neck was significantly higher in the ABT group (0.97\%) than in the Non-ABT group $(-2.19 \%$; $\mathrm{p}=0.026)$. Whereas, no significant difference in $\% \triangle \mathrm{BMD}$ at the lumbar spine was observed between groups (ABT, $-0.40 \%$; Non-ABT, $-1.67 \%$; $\mathrm{p}=0.524$ ). No significant differences were observed in changes to uNTx or BAP. ABT treatment was significantly associated with increased BMD at the femoral neck (odds ratio (OR), 8.84; 
95\%CI, 1.08-72.4; p=0.04), and baseline lumbar osteoarthritis was significantly associated with BMD at the lumbar spine (OR, 2.97; 95\%CI, 1.23-7.13; $\mathrm{p}=0.02)$.

Conclusion: The efficacy of ABT for increasing BMD at the femoral neck was superior to that of other bDMARDs. ABT may offer good efficacy for improving BMD at the femoral neck in patients with RA. 


\section{INTRODUCTION}

Patients with rheumatoid arthritis (RA) are known to be at higher risk for disorders of bone metabolism, and bone mineral density (BMD) is frequently lower in patients with RA than in healthy individuals [1]. Osteoporosis is frequently observed in patients with RA and represents an important risk for fragile fracture, increasing the risk of hip and vertebral fractures by about two- to six-fold in patients with RA compared to controls [2-4]. In patients with RA, bone metabolism is affected by some unexpected factors. In addition to primary osteoporosis with increasing age, patients with RA encounter three major conditions that contribute to osteoporosis. First, inflammation results in peri-articular osteoporosis around inflamed joints [5]. Second, oral glucocorticoids are known to lead to generalized osteoporosis [6,7], but are frequently used in the treatment of RA to suppress inflammation. Finally, inactivity resulting from joint destruction and disability frequently induces systemic osteoporosis.

Important influences on bone metabolism in patients with RA have been reported to include disease activity [8], history of osteoporosis treatment [9], and prednisolone (PSL) use [10]. We have previously clarified that decreasing PSL dose has an important effect in improving bone metabolic markers (BMMs) in patients with RA [10]. Whether biological disease-modifying anti-rheumatic drugs (bDMARDs) improve 
bone metabolism in patients with RA remains controversial [11-14]. Use of bDMARDs is reportedly effective for improving BMMs. However, the effects of tumor necrosis factor (TNF) inhibitors on BMD are not prominent. TNF inhibitors maintained BMD at the lumbar spine and hip for 6 months, but was unable to increase BMD significantly compared to baseline [14]. We also reported that the effect of TNF inhibitors on BMD in patients with RA was limited and reducing the dosage of glucocorticoids was effective for preserving bone metabolism [9].

Abatacept (ABT), a fusion protein of cytotoxic T lymphocyte-associated antigen-4 (CTLA-4) and immunoglobulin G1, selectively modulates the CD80/CD86:CD28 costimulatory signal required for full T-cell activation [15]. ABT is an effective treatment for patients with RA, according to both clinical trials [16-18] and practice [19]. The efficacy of ABT for improving disease activity and preventing joint destruction was similar to that of TNF inhibitor in the AMPLE study [18]. CTLA-4 has recently been reported to inhibit osteoclast differentiation by inducing the enzyme indoleamine 2,3-dioxygenase (IDO)/tryptophan pathway [20]. In a mouse model, CTLA-4 promoted Wnt-10b production and bone formation [21]. ABT may be effective for not only suppressing bone resorption, but also improving bone formation.

The degree to which ABT affects bone metabolism in patients with RA has yet to be 
clarified. No prospective comparative studies have directly investigated the effects of each bDMARD on bone metabolism. The present prospective, comparative study investigated the influence of ABT and other bDMARDs on BMD and BMMs in patients with RA in order to clarify the effects of ABT on bone metabolism.

\section{MATERIALS AND METHODS}

\section{Patients}

Eligible patients were $>20$ years old, fulfilled the ACR1987 classification criteria [22] or 2010 ACR/EULAR classification criteria [23], had been treated for at least 3 months, and required treatment with bDMARDs, including switching. Patients who were pregnant, breastfeeding, had an active infection, or had a significant concomitant disease were excluded. The protocol of this study was approved by the institutional review board of Osaka City University Medical School (ethics approval number: 2027) and conducted in accordance with the ethical standards outlined in the Declaration of Helsinki. All patients provided written informed consent prior to participation.

\section{Study design}

We initiated a prospective, comparative non-randomized study (AIRTIGHT: Can abatacept induce immunological Remission under Tight control in rheumatoid arthritis 
patient?) to investigate the effects of ABT and other bDMARDs on bone metabolism.

Consecutive RA patients scheduled to receive bDMARDs were recruited for this study until the number of ABT groups reached 50 (See statistical analysis section). The treatment decision was made by each physician independently and without randomizing.

This study was registered with the UMIN Clinical Trials Registry [http://www.umin.ac.jp/ctr/] (UMIN000005570) and conducted at three hospitals in Japan between May 2011 and May 2014. One of the primary endpoints was the change in BMD at 48 weeks. Secondary endpoints were variations in BMMs and disease activity score in 28 joints using erythrocyte sedimentation rate (DAS28-ESR) [24]. ABT was administered intravenously every 4 weeks for a total of 48 weeks. Other bDMARDs were also administered in accordance with the respective drug package inserts. Patients were divided into ABT and Non-ABT groups without randomization, then BMD and BMMs were evaluated over the course of 48 weeks.

\section{Assessment}

This study was an investigator-initiated trial and the treating physicians and patients were not blinded to the prescribed medications, but clinical parameters and outcomes were assessed during the treatment period by trained evaluators blinded to the treatment 
assignment and clinical response of each patient. BMDs at the femoral neck and lumbar spine (L2-4, anteroposterior view) was evaluated by dual-energy X-ray absorptiometry (DXA) using a QDR 4500 system (Hologic, Waltham, MA) at baseline and at 48 weeks. The percentage change in $\mathrm{BMD}(\% \Delta \mathrm{BMD})$ was calculated to compare the $\mathrm{ABT}$ and Non-ABT groups. As BMMs, urinary levels of cross-linked N-telopeptide of type I collagen (uNTx) and bone-specific alkaline phosphatase (BAP) were used as markers of bone resorption and bone formation, respectively, at baseline and 48 weeks. Disease activity was evaluated using the DAS28-ESR [24] at baseline and every 4 weeks for a total 48 weeks.

\section{Statistical analysis}

A sample of 165 patients (50 patients in ABT group, 115 patients in Non-ABT group) was calculated as necessary to provide at least $80 \%$ power for detecting a significant ( $\mathrm{p}$ $<0.05)$ difference in mean change of BMD and BMM at 1 year between groups. We asked all patients who would receive bDMARDS to participate in this study until the number of ABT groups reaches 50. All data are presented as mean \pm standard deviation (SD) or median (interquartile range), as indicated. The significance of differences in baseline characteristics between groups was tested using the unpaired t-test, Mann-Whitney $U$ test and Fisher's exact probability test. The $\% \Delta B M D$ at the femoral 
neck and lumbar spine were compared using the unpaired t-test and changes in uNTx and BAP were compared using the Mann-Whitney $U$ test between ABT and Non-ABT groups. The significance of changes in DAS28-ESR scores at each time point was tested using the paired t-test. Mean DAS28-ESR scores in both groups were compared using the unpaired t-test. Multivariate logistic regression analysis was performed to investigate factors potentially associated with increased BMD at the femoral neck and lumbar spine. We calculated odds ratios (ORs) and 95\% confidence intervals (CI) with adjustment by several confounders including age. Increases in BMD at the femoral neck and lumbar spine were defined as an increase in BMD above the least significant change (LSC) [25]. Since coefficients of variation of BMD values at the femoral neck and lumbar spine at our facility were $0.4 \%$ and $2.1 \%$, LSC was calculated as $1.1 \%$ and $5.8 \%$. Values of $\mathrm{p}<0.05$ were considered significant. All statistical analyses were performed using IBM SPSS Statistics version 22 (IBM, Armonk, NY).

\section{RESULTS}

\section{Patient characteristics}

A total of 169 patients with RA were screened initially and 165 eligible patients were finally enrolled in the AIRTIGHT study. Patients were divided into two groups: 50 
patients who received $\mathrm{ABT}$ (ABT group), and 115 who received other bDMARDs (Non-ABT group). The Non-ABT group received infliximab (IFX) $(\mathrm{n}=15)$, etanercept $($ ETN $)(n=22)$, adalimumab (ADA) $(n=38)$, tocilizumab $($ TCZ $)(n=26)$, or golimumab $(\mathrm{GLM})(\mathrm{n}=14)$. As shown in Fig. $1,36 \%$ and $26.9 \%$ of patients in the ABT and Non-ABT groups discontinued treatment before completing the 48-week regimen, respectively. No significant differences in reasons for discontinuation were seen between groups. The percentage continuing treatment was $64.0 \%$ in the ABT group and $73.1 \%$ in the Non-ABT group, showing no significant difference $(\mathrm{p}=0.387)$. Among those patients, DXA data were collected and analyzed for 30 patients from the ABT group and 78 patients from the Non-ABT group.

Patient demographics and clinical characteristics at baseline are summarized in Table 1. Rate of previous usage of biologics was significantly higher in the ABT group (50.0\%) than in the Non-ABT group $(27.8 \% ; \mathrm{p}=0.008)$. On the other hand, the rate of methotrexate (MTX) usage was significantly lower in the ABT group (72.0\%) than in the Non-ABT group $(86.1 \% ; \mathrm{p}=0.047)$. In all patients, median disease duration was 7 years and mean DAS28-ESR was 5.25. All patients showed established RA with high disease activity. No significant differences in usage rate and dosage of prednisolone, osteoporosis treatment rate, bisphosphonate usage rate, BMD at the lumbar spine and 
femoral neck, or BMMs at baseline were identified between groups. The details of osteoporosis treatment were 13 cases by bisphosphonate and 5 cases by active vitamin $\mathrm{D}$ in $\mathrm{ABT}$ group, and 41 cases by bisphosphonate, 4 cases by active vitamin $\mathrm{D}, 3$ cases by selective estrogen receptor modulators, and 2 cases by teriparatide in Non-ABT group.

\section{BMD}

The $\% \triangle B M D$ is shown in Fig. 2. The $\% \triangle B M D$ at the femoral neck was significantly higher in the ABT group $(0.97 \pm 8.63 \%)$ than in the Non-ABT group $(-2.19 \pm 5.49 \%$; $\mathrm{p}=0.026$ ) (Fig. 2A). On the other hand, no significant difference in the $\% \Delta B M D$ at the lumbar spine was observed between groups (ABT, $-0.40 \pm 4.31 \%$; Non-ABT, $-1.67 \pm$ 5.07\%; p=0.524) (Fig. 2B).

Multivariate logistic regression analysis (Table 2) revealed that the most significant influence on increased BMD at the femoral neck was ABT use (OR, 8.84; 95\%CI, 1.08-72.4; $\mathrm{p}=0.04)$. Baseline osteoporosis treatment, decreased PSL, and change in DAS28-ESR were not identified as factors influencing increased BMD. For increased BMD at the lumbar spine, baseline lumbar osteoarthritis on radiography was identified as an influencing factor $(\mathrm{OR}, 2.97 ; 95 \% \mathrm{CI}, 1.23-7.13 ; \mathrm{p}=0.02)$.

Among patients without lumbar osteoarthritis, a significant correlation was evident 
between $\% \Delta \mathrm{BMD}$ at the lumbar spine and femoral neck $(\mathrm{r}=0.452, \mathrm{p}=0.001)$, whereas patients with lumbar osteoarthritis showed no such correlation $(\mathrm{r}=0.083, \mathrm{p}=0.612)$.

\section{BMMs}

Changes in BMMs are shown in Fig. 3. The $\Delta \mathrm{uNTx}$ was negative in both the ABT group $(-3.55[-21.23,3.83] \mathrm{nmol} \mathrm{BCE} / \mathrm{mmol} \mathrm{Cr})$ and the Non-ABT group (-0.11 [-15.21, 10.75] nmol BCE/mmol Cr). No significant difference was evident between groups $(\mathrm{p}=0.370)($ Fig. 3A). With $\triangle \mathrm{BAP}$ as a bone formation marker, no significant differences were observed between groups (ABT, $-0.15[-20.65,3.35] \mu \mathrm{g} / \mathrm{L}$; Non-ABT, 1.52 $[-14.92,10.21] \mu \mathrm{g} / \mathrm{L} ; \mathrm{p}=0.276)$ (Fig. 3B).

\section{Disease activity}

Details of disease activity are shown in Fig. 4. From 12 weeks, DAS28-ESR was significantly improved compared with baseline in both groups. DAS28-ESR was significantly higher in the ABT group than in the Non-ABT group from week 12 of the observational period. At 48 weeks, DAS28-ESR of the ABT group still had not reached that of the Non-ABT group (ABT, $4.13 \pm 1.31$; Non-ABT, $3.46 \pm 1.07 ; \mathrm{p}=0.008$ ).

\section{DISCUSSION}

This study is the first to indicate that $\mathrm{ABT}$ is effective for $\% \triangle \mathrm{BMD}$ at the femoral neck 
compared to Non-ABT. BMD at the lumbar spine tended to decrease less from baseline in ABT than in Non-ABT, but the result was not significant. ABT decreased NTx and increased BAP from baseline. The effects on BMMs were not significantly better in the ABT group than in the Non-ABT group.

In this study, ABT increased BMD at the femoral neck and maintained the lumbar spine, and offered comparable efficacy to other bDMARDs. The efficacy of ABT for bone metabolism in older patients (70 years old or older) showed the same tendency as the whole. However, there was no significant difference in the $\% \Delta B M D$ at the femoral neck between groups (ABT: $0.8 \pm 5.6$, Non-ABT: $-2.0 \pm 5.5, \mathrm{P}=0.209$ ). We supposed that we could not show a significant difference in older patients as the number of elderly patients was small (ABT: 9 cases, Non-ABT: 23 cases).

We have previously reported the effects of TNF inhibitors on BMD in patients with RA as limited [9]. Conversely, some researchers have reported that anti-IL-6R (interleukin-6 receptor) increased BMD at the lumbar spine and femoral neck in patients with RA who showed osteopenia at baseline [13]. The mechanisms by which ABT increased BMD at the femoral neck remain unclear. CTLA-4 immunoglobulin has been reported to promote Wnt-10b production and bone formation [21]. The fact that we could not detect any significant effects of ABT on BMMs may be partially attributable 
to the small sample size.

The treatment of $\mathrm{ABT}$ was found to significantly influence the increase in BMD at the femoral neck on multivariate logistic regression analysis. Two main reasons for this result are apparent. First, patients were treated using not only ABT, but also agents used for treating osteoporosis, including bisphosphonate. ABT might manifest synergistic effects in use with anti-osteoporosis agents. Second, osteoporosis treatments in some patients were newly started because their BMD was measured according to the protocol of this prospective study. On the other hand, lumbar osteoarthritis was the only factor associated with increased BMD at the lumbar spine. BMD at the lumbar spine is highly influenced by osteoarthritis, occult vertebral fracture and degenerative changes, particularly in elderly patients [26]. In our study, patients with lumbar osteoarthritis showed no correlation between $\% \Delta \mathrm{BMD}$ at the lumbar spine and femoral neck. Change in PSL dosage and osteoporosis treatment were not found to be significant factors influencing changes in BMD at either the femoral neck or lumbar spine in the present study. This might be due to the relatively small changes in PSL dosage and low percentage of patients receiving osteoporosis treatment from baseline to 48 weeks. Patients treated with ABT showed comparable efficacy for BMD to those treated with Non-ABT. However, disease activity scores decreased less with ABT than with 
Non-ABT and did not catch up to those in the Non-ABT group during the 48 weeks. At baseline, the rate of previous usage of biologics was significantly higher in the ABT group (50.0\%), while the MTX usage rate was significantly lower in the ABT group (72.0\%) than in the Non-ABT group. Patients treated with ABT had a more disadvantageous background than patients treated with Non-ABT. A discrepancy was seen between effects on bone metabolism and disease activity in patients treated with ABT.

Some limitations must be considered when interpreting the findings of this study. First, eligible patients were not divided randomly and our data included some degree of selection bias. To reveal the effects of $\mathrm{ABT}$ on $\mathrm{BMD}$ more clearly, a prospective randomized control study is needed. Second, the sample size was too small to compare the effects of each bDMARD on BMD. Third, this study was performed under real clinical conditions and the discontinuation rate was high in both groups. Discontinued patients who switched bDMARDs and experienced adverse events need to be included to clarify the efficacy of bDMARDs in improving BMD.

The present findings can be interpreted as indicating that ABT might offer better efficacy for increase BMD at the femoral neck than Non-ABT. Patients with RA have a higher risk of fragile fracture compared with healthy subjects [2-4] and osteoporosis is 
the one of the most important complications [27]. If ABT can improve bone metabolism in addition to controlling disease activity and suppressing radiographic progression, the risk of fragile fracture due to osteoporosis may gradually decrease under ABT treatment.

In conclusions, the efficacy of ABT in improving bone metabolism in patients with RA was comparable to that of other bDMARDs in this prospective, comparative study. Treatment of ABT was a significant influence on increased BMD at the femoral neck. ABT may offer good efficacy for improving bone mineral density at the femoral neck in patients with RA. 


\section{REFERENCES}

1. Haugeberg G, Uhlig T, Falch JA, Halse JI, Kvien TK (2000) Bone mineral density and frequency of osteoporosis in female patients with rheumatoid arthritis: results from 394 patients in the Oslo County Rheumatoid Arthritis register. Arthritis Rheum 43 (3):522-530. doi:10.1002/1529-0131(200003)43:3<522::AID-ANR7>3.0.CO;2-Y

2. Peel NF, Moore DJ, Barrington NA, Bax DE, Eastell R (1995) Risk of vertebral fracture and relationship to bone mineral density in steroid treated rheumatoid arthritis. Ann Rheum Dis 54 (10):801-806

3. van Staa TP, Geusens P, Bijlsma JW, Leufkens HG, Cooper C (2006) Clinical assessment of the long-term risk of fracture in patients with rheumatoid arthritis. Arthritis Rheum 54 (10):3104-3112. doi:10.1002/art.22117

4. Wright NC, Lisse JR, Walitt BT, Eaton CB, Chen Z, Women's Health Initiative I (2011) Arthritis increases the risk for fractures--results from the Women's Health Initiative. J Rheumatol 38 (8):1680-1688. doi:10.3899/jrheum.101196

5. Braun T, Schett G (2012) Pathways for bone loss in inflammatory disease. Curr Osteoporos Rep 10 (2):101-108. doi:10.1007/s11914-012-0104-5

6. Van Staa TP, Leufkens HG, Abenhaim L, Zhang B, Cooper C (2000) Use of oral 
corticosteroids and risk of fractures. J Bone Miner Res 15 (6):993-1000.

doi:10.1359/jbmr.2000.15.6.993

7. van Staa TP, Leufkens HG, Cooper C (2002) The epidemiology of

corticosteroid-induced osteoporosis: a meta-analysis. Osteoporos Int 13

(10):777-787. doi:10.1007/s001980200108

8. Vis M, Guler-Yuksel M, Lems WF (2013) Can bone loss in rheumatoid arthritis be prevented? Osteoporos Int 24 (10):2541-2553. doi:10.1007/s00198-013-2334-5

9. Okano T, Koike T, Tada M, Sugioka Y, Mamoto K, Wakitani S, Nakamura H (2014)

The limited effects of anti-tumor necrosis factor blockade on bone health in patients with rheumatoid arthritis under the use of glucocorticoid. J Bone Miner Metab 32 (5):593-600. doi:10.1007/s00774-013-0535-9

10. Tada M, Inui K, Sugioka Y, Mamoto K, Okano T, Koike T, Nakamura H (2016) Reducing glucocorticoid dosage improves serum osteocalcin in patients with rheumatoid arthritis-results from the TOMORROW study. Osteoporos Int 27 (2):729-735. doi:10.1007/s00198-015-3291-y

11. Haugeberg G, Helgetveit KB, Forre O, Garen T, Sommerseth H, Proven A (2014) Generalized bone loss in early rheumatoid arthritis patients followed for ten years in the biologic treatment era. BMC Musculoskelet Disord 15:289. 
doi:1471-2474-15-289 [pii]

$10.1186 / 1471-2474-15-289$

12. Kawai VK, Stein CM, Perrien DS, Griffin MR (2012) Effects of anti-tumor necrosis factor alpha agents on bone. Curr Opin Rheumatol 24 (5):576-585. doi:10.1097/BOR.0b013e328356d212

13. Kume K, Amano K, Yamada S, Kanazawa T, Ohta H, Hatta K, Kuwaba N (2014) The effect of tocilizumab on bone mineral density in patients with methotrexate-resistant active rheumatoid arthritis. Rheumatology (Oxford) 53 (5):900-903. doi:ket468 [pii]

10.1093/rheumatology/ket468

14. Seriolo B, Paolino S, Sulli A, Ferretti V, Cutolo M (2006) Bone metabolism changes during anti-TNF-alpha therapy in patients with active rheumatoid arthritis. Ann N Y Acad Sci 1069:420-427. doi:1069/1/420 [pii]

10.1196/annals. 1351.040

15. Moreland LW, Alten R, Van den Bosch F, Appelboom T, Leon M, Emery P, Cohen S, Luggen M, Shergy W, Nuamah I, Becker JC (2002) Costimulatory blockade in patients with rheumatoid arthritis: a pilot, dose-finding, double-blind, placebo-controlled clinical trial evaluating CTLA-4Ig and LEA29Y eighty-five 
days after the first infusion. Arthritis Rheum 46 (6):1470-1479.

doi:10.1002/art.10294

16. Emery P, Burmester GR, Bykerk VP, Combe BG, Furst DE, Barre E, Karyekar CS, Wong DA, Huizinga TW (2015) Evaluating drug-free remission with abatacept in early rheumatoid arthritis: results from the phase $3 \mathrm{~b}$, multicentre, randomised, active-controlled AVERT study of 24 months, with a 12-month, double-blind treatment period. Ann Rheum Dis 74 (1):19-26. doi:annrheumdis-2014-206106 [pii]

10.1136/annrheumdis-2014-206106

17. Schiff M, Keiserman M, Codding C, Songcharoen S, Berman A, Nayiager S, Saldate C, Li T, Aranda R, Becker JC, Lin C, Cornet PL, Dougados M (2008) Efficacy and safety of abatacept or infliximab vs placebo in ATTEST: a phase III, multi-centre, randomised, double-blind, placebo-controlled study in patients with rheumatoid arthritis and an inadequate response to methotrexate. Ann Rheum Dis 67 (8):1096-1103. doi:ard.2007.080002 [pii]

10.1136/ard.2007.080002

18. Schiff M, Weinblatt ME, Valente R, van der Heijde D, Citera G, Elegbe A, Maldonado M, Fleischmann R (2014) Head-to-head comparison of subcutaneous 
abatacept versus adalimumab for rheumatoid arthritis: two-year efficacy and safety findings from AMPLE trial. Ann Rheum Dis 73 (1):86-94.

doi:annrheumdis-2013-203843 [pii]

10.1136/annrheumdis-2013-203843

19. Takahashi N, Kojima T, Kaneko A, Kida D, Hirano Y, Fujibayashi T, Yabe Y, Takagi H, Oguchi T, Miyake H, Kato T, Watanabe T, Hayashi M, Kanayama Y, Funahashi K, Asai S, Yoshioka Y, Takemoto T, Terabe K, Asai N, Ishiguro N (2015) Longterm efficacy and safety of abatacept in patients with rheumatoid arthritis treated in routine clinical practice: effect of concomitant methotrexate after 24 weeks. J Rheumatol 42 (5):786-793. doi:jrheum.141288 [pii]

10.3899/jrheum.141288

20. Bozec A, Zaiss MM, Kagwiria R, Voll R, Rauh M, Chen Z, Mueller-Schmucker S, Kroczek RA, Heinzerling L, Moser M, Mellor AL, David JP, Schett G (2014) T cell costimulation molecules CD80/86 inhibit osteoclast differentiation by inducing the IDO/tryptophan pathway. Sci Transl Med 6 (235):235ra260. doi:6/235/235ra60 [pii]

10.1126/scitranslmed.3007764

21. Roser-Page S, Vikulina T, Zayzafoon M, Weitzmann MN (2014) 
CTLA-4Ig-induced T cell anergy promotes Wnt-10b production and bone formation in a mouse model. Arthritis Rheumatol 66 (4):990-999.

doi:10.1002/art.38319

22. Arnett FC, Edworthy SM, Bloch DA, McShane DJ, Fries JF, Cooper NS, Healey LA, Kaplan SR, Liang MH, Luthra HS, et al. (1988) The American Rheumatism Association 1987 revised criteria for the classification of rheumatoid arthritis. Arthritis Rheum 31 (3):315-324

23. Aletaha D, Neogi T, Silman AJ, Funovits J, Felson DT, Bingham CO, 3rd, Birnbaum NS, Burmester GR, Bykerk VP, Cohen MD, Combe B, Costenbader KH, Dougados M, Emery P, Ferraccioli G, Hazes JM, Hobbs K, Huizinga TW, Kavanaugh A, Kay J, Kvien TK, Laing T, Mease P, Menard HA, Moreland LW, Naden RL, Pincus T, Smolen JS, Stanislawska-Biernat E, Symmons D, Tak PP, Upchurch KS, Vencovsky J, Wolfe F, Hawker G (2010) 2010 Rheumatoid arthritis classification criteria: an American College of Rheumatology/European League Against Rheumatism collaborative initiative. Arthritis Rheum 62 (9):2569-2581. doi:10.1002/art.27584

24. Prevoo ML, van 't Hof MA, Kuper HH, van Leeuwen MA, van de Putte LB, van Riel PL (1995) Modified disease activity scores that include twenty-eight-joint 
counts. Development and validation in a prospective longitudinal study of patients with rheumatoid arthritis. Arthritis Rheum 38 (1):44-48

25. Bonnick SL, Johnston CC, Jr., Kleerekoper M, Lindsay R, Miller P, Sherwood L, Siris E (2001) Importance of precision in bone density measurements. J Clin Densitom 4 (2):105-110

26. Steiger P, Cummings SR, Black DM, Spencer NE, Genant HK (1992) Age-related decrements in bone mineral density in women over 65. J Bone Miner Res 7 (6):625-632. doi:10.1002/jbmr.5650070606

27. Deodhar AA, Woolf AD (1996) Bone mass measurement and bone metabolism in rheumatoid arthritis: a review. Br J Rheumatol 35 (4):309-322 
Table 1: Patient demographics and clinical characteristics at baseline

\begin{tabular}{|c|c|c|c|}
\hline Parameter & $\operatorname{ABT}(\mathbf{n}=50)$ & Non-ABT $(n=115)$ & $p$ \\
\hline Age, years & $61.3 \pm 13.2$ & $60.9 \pm 13.2$ & 0.876 \\
\hline Disease duration, years & $8.5[3.0,19.0]$ & $6.0[1.8,15.0]$ & 0.214 \\
\hline Female, \% & 86.0 & 83.5 & 0.818 \\
\hline Previous use of biologics, $\%$ & 50.0 & 27.8 & 0.008 \\
\hline $\mathrm{CRP}, \mathrm{mg} / \mathrm{dl}$ & $1.8 \pm 2.5$ & $2.0 \pm 2.5$ & 0.733 \\
\hline DAS28-ESR & $5.38 \pm 1.41$ & $5.17 \pm 1.31$ & 0.356 \\
\hline Use of MTX, \% & 72.0 & 86.1 & 0.047 \\
\hline MTX dosage, mg/week* & $8.9 \pm 2.6$ & $9.9 \pm 3.1$ & 0.076 \\
\hline Use of PSL, \% & 42.0 & 51.3 & 0.311 \\
\hline PSL dosage, mg/day* & $3.8 \pm 1.8$ & $4.4 \pm 2.9$ & 0.377 \\
\hline Anti CCP antibody positive, $\%$ & 81.6 & 87.2 & 0.342 \\
\hline Rheumatoid factor positive, $\%$ & 69.3 & 78.2 & 0.239 \\
\hline mHAQ & $0.81 \pm 0.66$ & $0.77 \pm 0.65$ & 0.852 \\
\hline mTSS & $36.5[15.4,109]$ & $37.5[13.6,123]$ & 0.864 \\
\hline Lumbar spine BMD, $\mathrm{g} / \mathrm{cm}^{2}$ & $0.871 \pm 0.143$ & $0.900 \pm 0.161$ & 0.386 \\
\hline
\end{tabular}




$\begin{array}{lllr}\text { Femoral neck BMD, g/cm }{ }^{2} & 0.594 \pm 0.114 & 0.617 \pm 0.126 & 0.375 \\ \text { uNTx, nmolBCE/mmolCr } & 51.3[27.8,85.0] & 40.5[30.3,60.2] & 0.161 \\ \text { BAP, } \mu \mathrm{g} / \mathrm{L} & 17.8[12.4,22.8] & 15.7[11.6,22.7] & 0.478 \\ \text { On osteoporosis treatment, \% } & 36.0 & 43.4 & 0.387 \\ & & & 0.280\end{array}$

Mean \pm standard deviation (SD) or median [25th, 75 th percentile],

ABT, abatacept; CRP, C-reactive protein; DAS, disease activity score; ESR, erythrocyte sedimentation rate; MTX, methotrexate; PSL, prednisolone; CCP, cyclic citrullinated peptide; mHAQ, modified health assessment questionnaire; mTSS, modified total Sharp score; BMD, bone mineral density; uNTx, urinary cross-linked N-telopeptide of type I collagen; BAP, bone-specific alkaline phosphate; * Calculated only for the relevant patients. 
Table 2: Logistic multiple regression analysis of increases in BMD at the femoral neck and lumbar spine

\begin{tabular}{|c|c|c|c|c|c|c|}
\hline & \multicolumn{3}{|c|}{ Femoral neck } & \multicolumn{3}{|c|}{ Lumbar spine } \\
\hline & $\mathrm{OR}^{\dagger}$ & $95 \% \mathrm{CI}$ & $p$ & $\mathrm{OR}^{\dagger}$ & $95 \% \mathrm{CI}$ & $p$ \\
\hline ABT use $(0$, no; 1, yes $)$ & 8.84 & $1.08-72.4$ & 0.04 & 0.99 & $0.36-2.75$ & 0.98 \\
\hline Steinbrocker class & 0.54 & $0.15-1.89$ & 0.33 & 1.23 & $0.63-2.41$ & 0.53 \\
\hline Baseline mHAQ & 1.86 & $0.41-8.52$ & 0.42 & 0.91 & $0.41-1.99$ & 0.81 \\
\hline OP treatment $(0$, no; 1, yes $)$ & 0.39 & $0.04-3.86$ & 0.42 & 0.82 & $0.34-1.99$ & 0.65 \\
\hline Change of PSL & 0.80 & $0.43-1.49$ & 0.47 & 0.86 & $0.66-1.12$ & 0.27 \\
\hline Change of DAS28-ESR & 1.37 & $0.63-2.97$ & 0.43 & 1.02 & $0.64-1.64$ & 0.94 \\
\hline Lumbar OA $(0$, no; 1 , yes $)$ & 3.50 & $0.59-20.6$ & 0.17 & 2.97 & $1.23-7.13$ & 0.02 \\
\hline
\end{tabular}

$\dagger$ ORs were adjusted by age. 
$\mathrm{BMD}$, bone mineral density; OR, odds ratio; CI, confidence interval; ABT, abatacept; mHAQ, modified health assessment questionnaire; OP, osteoporosis; PSL, prednisolone; DAS, disease activity score; ESR, erythrocyte sedimentation rate; OA, osteoarthritis 


\section{FIGURE LEGENDS}

Figure. 1 Patient disposition and reasons for discontinuation. 


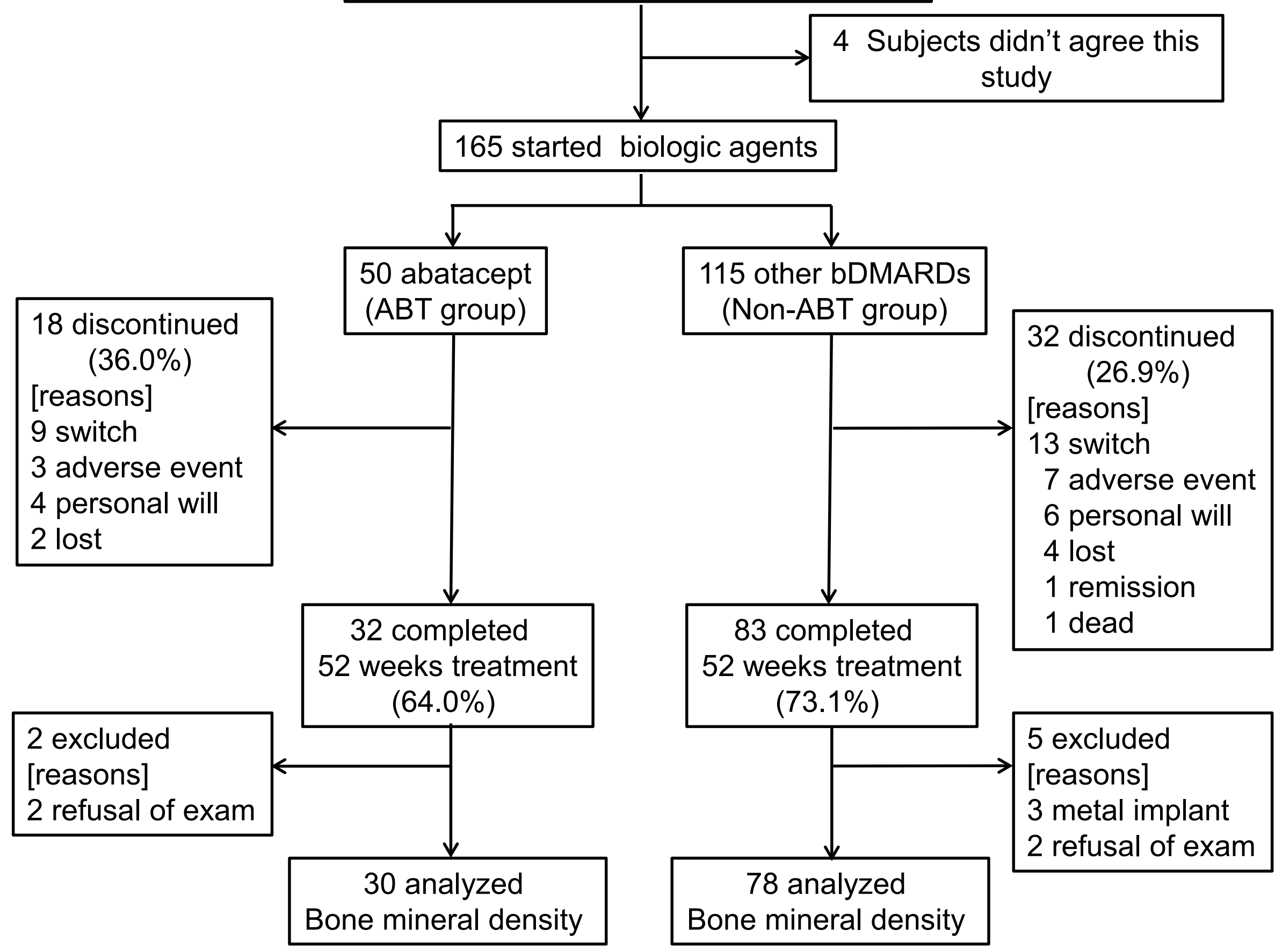


Figure. 2 Mean ( \pm standard deviation $[\mathrm{SD}])$ percentage change in bone mineral density $(\% \Delta \mathrm{BMD})$ at the femoral neck $(\mathrm{A})$ and lumbar spine (B). Results for abatacept (ABT) and Non-ABT were compared using the unpaired t-test. 

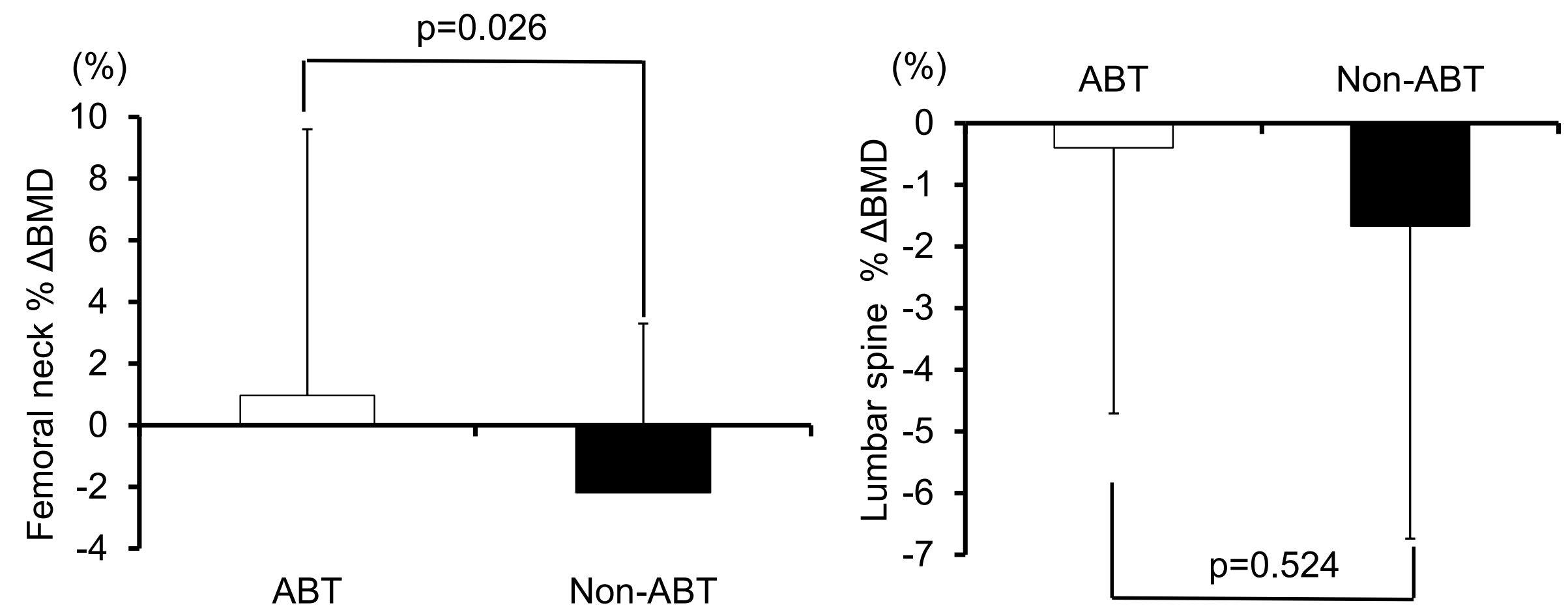

(A)

(B) 
Figure. 3 Median [25th, 75th percentile] change in urinary cross-linked N-telopeptide of type I collagen $(\Delta \mathrm{uNTx})$ as a bone resorption marker $(\mathrm{A})$ and bone-specific alkaline phosphatase $(\triangle \mathrm{BAP})$ as a bone formation marker $(\mathrm{B})$. Results for abatacept $(\mathrm{ABT})$ and Non-ABT were calculated using the Mann-Whitney U test. 

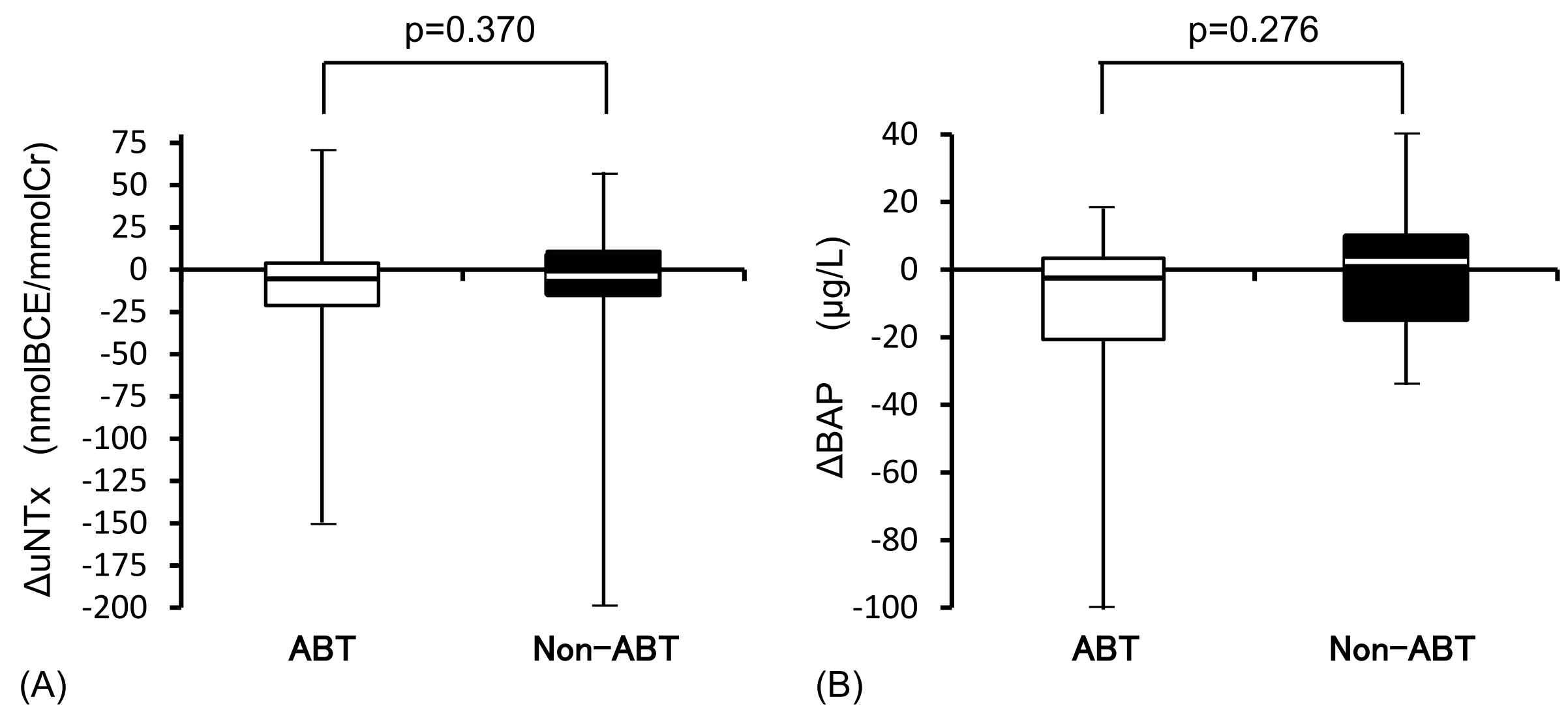
Figure. 4 Mean ( \pm standard deviation $[S D]$ ) change in DAS28-ESR from baseline.

${ }^{*} \mathrm{p}<0.05$ change from baseline for both abatacept (ABT) and Non-ABT by paired $t$-test.

$\dagger \mathrm{p}<0.05$ compared with ABT by unpaired $t$-test. 


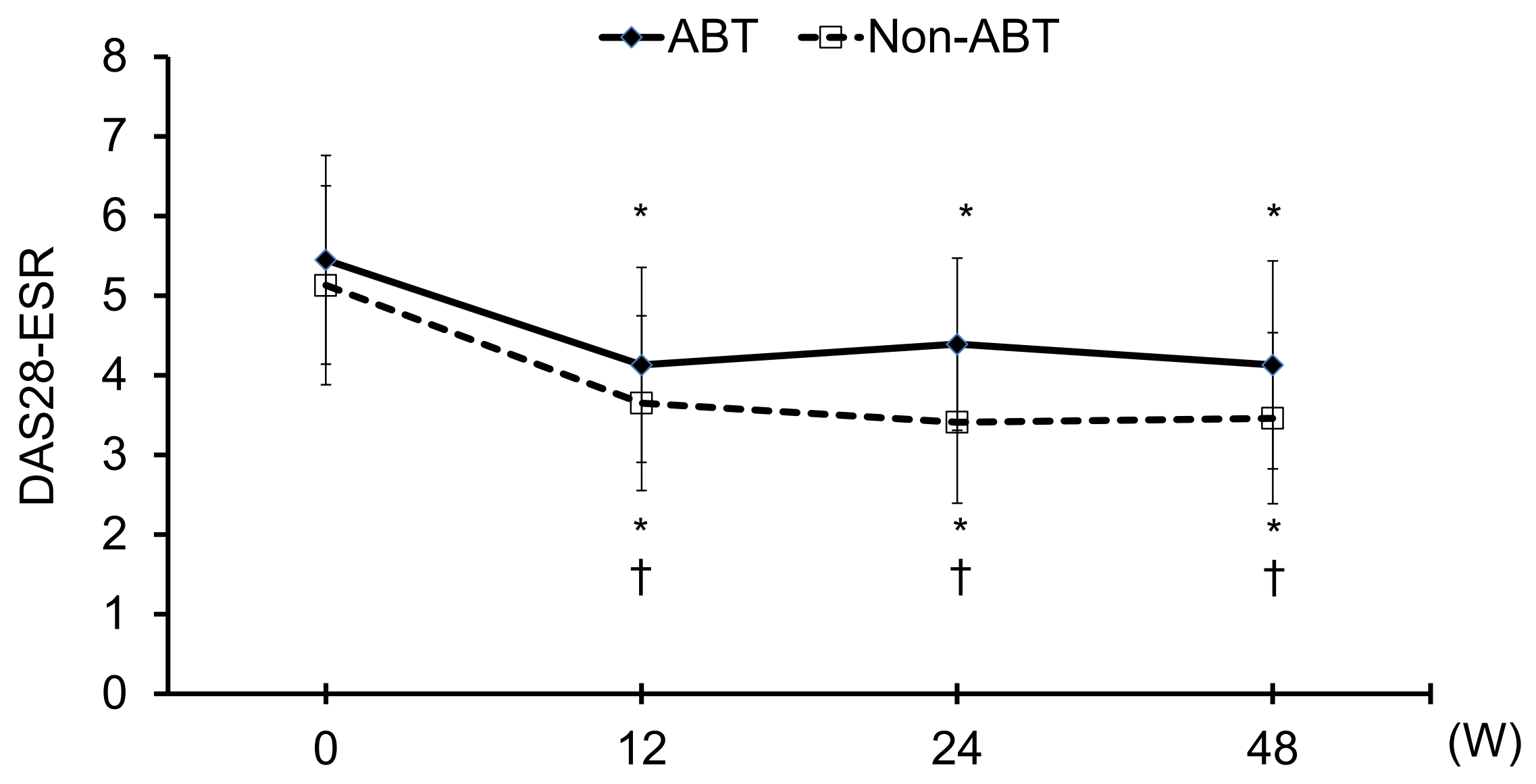

